\title{
ON TRANSLATION-BOUNDED MEASURES
}

\section{A. P. ROBERTSON and M. L. THORNETT}

(Received 20 February 1983)

Communicated by R. L. Tweedie

\begin{abstract}
It is shown that a positive measure $\mu$ on the Borel subsets of $\mathbf{R}^{k}$ is translation-bounded if and only if the Fourier transform of the indicator function of every bounded Borel subset of $\mathbf{R}^{k}$ belongs to $L^{2}(\mu)$. 1980 Mathematics subject classification (Amer. Math. Soc.): primary $60 \mathrm{G} \mathrm{10}$; secondary 28 A 33, 42 B 10.
\end{abstract}

\section{Introduction}

We shall be considering non-negative measures defined on the class $\mathscr{B}$ of Borel subsets of $\mathbf{R}^{k}$, taking finite values on the subclass $\mathscr{S}_{0}$ of bounded Borel sets; for convenience these will be called Borel measures. A Borel measure $\mu$ is called translation-bounded if, for every $A \in \Re_{0}$,

$$
\sup \left\{\mu(A+x): x \in \mathbf{R}^{k}\right\}<\infty .
$$

It is clearly sufficient that this property hold for some $A_{0}$ with non-empty interior, for by a compactness argument any other $A \in \mathscr{B}_{0}$ can be covered by a finite union of translates of $A_{0}$.

As in [3], we shall use the same notation for a set $A$ and its indicator function; thus $A(x)=1$ if $x \in A$ and $A(x)=0$ otherwise. For each $A \in \mathscr{G}_{0}$, its Fourier transform $\hat{A}$ is defined for all $\xi \in \mathbf{R}^{k}$ by

$$
\hat{A}(\xi)=\int_{\mathbf{R}^{k}} A(x) e^{i x \cdot \xi} d x,
$$

where $x . \xi$ denotes the canonical inner product in $\mathbf{R}^{k}$.

(ㄷ) 1984 Australian Mathematical Society $0263-6115 / 84 \$ A 2.00+0.00$ 
It is shown in [3] that if $\mu$ is translation-bounded, then $\hat{A} \in L^{2}(\mu)$ for every $A \in \mathscr{B}_{0}$. The purpose of this note is to prove the converse and thus establish the following result.

THEOREM. A non-negative Borel measure $\mu$ on $\mathbf{R}^{k}$ is translation-bounded if and only if $\hat{A} \in L^{2}(\mu)$ for every bounded Borel subset $A$ of $\mathbf{R}^{k}$.

\section{Proof of the theorem}

We are given that $\mu$ is a non-negative Borel measure, finite on bounded sets, for which $\hat{A} \in L^{2}(\mu)$ for each $A \in \mathscr{B}_{0}$.

Take any subset $I \in \mathscr{B}_{0}$ with nonempty interior and denote by $B(I)$ the space of bounded Borel-measurable (complex-valued) functions on $I$, with the supremum norm. The indicator functions of Borel subsets of $I$ form a subset $X(I)$, generating the dense vector subspace $S(I)$ of $B(I)$ consisting of the simple functions on $I$.

Every function $f \in B(I)$ has a Fourier transform $\hat{f}=T(f)$; the main part of the proof is to show the continuity of $T$.

LEMMA. Under the hypothesis of the theorem, $T$ is a continuous linear transformation from $B(I)$ to $L^{2}(\mu)$; that is, there is a constant c such that

$$
\int|\hat{f}(\xi)|^{2} d \mu \leqslant c^{2} \sup \left\{|f(x)|^{2}: x \in I\right\} \quad \text { for all } f \in B(I) .
$$

Proof. Take any compact set $K_{1}$ in $\mathbf{R}^{k}$ and any $g \in L^{2}(\mu)$.

By hypothesis, $\hat{A} \in L^{2}(\mu)$ for each $A \in X(I)$ and so

$$
\nu_{1}(A)=\int_{K_{1}} \hat{A} \bar{g} d \mu
$$

is defined in $X(I)$. In fact, $\nu_{1}$ is a (complex-valued) measure on the Borel subsets of $I$. For $\nu_{1}$ is clearly finitely additive. Also, if $\left(A_{n}\right)$ is a sequence decreasing to the empty set, then

$$
\left|\nu_{1}\left(A_{n}\right)\right|^{2} \leqslant \int_{K_{1}}\left|\hat{A}_{n}\right|^{2} d \mu \cdot \int_{K_{1}}|\bar{g}|^{2} d \mu \leqslant\|g\|_{2}^{2} \mu\left(K_{1}\right)\left(\lambda\left(A_{n}\right)\right)^{2},
$$

where $\lambda$ denotes Lebesgue measure in $\mathbf{R}^{k}$. So $\nu_{1}\left(A_{n}\right) \rightarrow 0$ as $n \rightarrow \infty$ and therefore $\nu_{1}$ is countably additive. 
Now take a sequence of compact sets $K_{n}$ increasing to $\mathbf{R}^{k}$ and let $\nu_{n}$ be the corresponding measures. For each $A \in X(I)$,

$$
\lim _{n \rightarrow \infty} \nu_{n}(A)=\lim _{n \rightarrow \infty} \int_{K_{n}} \hat{A} \bar{g} d \mu=\int_{\mathbf{R}^{k}} \hat{A} \bar{g} d \mu=\nu(A)
$$

exists by hypothesis. Hence, by the theorem of Nikodým ([1] page 160, [2]) $\nu$ is a measure, which is therefore bounded on the Borel subsets of $I$. This shows that the set of $\hat{A}$ with $A \in X(I)$ is weakly bounded in $L^{2}(\mu)$ and hence, by the uniform boundedness theorem, it is norm-bounded:

$$
\sup \left\{\|\hat{A}\|_{2}: A \in X(I)\right\}<\infty .
$$

Now, since every $f \in S(I)$ with $0 \leqslant f(x) \leqslant 1$ on $I$ is a convex combination of elements of $X(I),\{\hat{f}: f \in S(I)\}$ is also norm-bounded in $L^{2}(\mu)$. Hence there is a constant $c$ such that

$$
\|\hat{f}\|_{2} \leqslant c\|f\|_{\infty} \text { for all } f \in S(I)
$$

Finally, any $f \in B(I)$ is the uniform limit of a sequence $\left(f_{n}\right)$ of functions of $S(I)$. By the continuity of $T$ on $S(I),\left(\hat{f}_{n}\right)$ converges in $L^{2}(\mu)$, but also $\left(\hat{f}_{n}\right)$ converges to $\hat{f}$ pointwise on $\mathbf{R}^{k}$. So

$$
\|\hat{f}\|_{2}=\lim _{n \rightarrow \infty}\left\|\hat{f}_{n}\right\|_{2} \leqslant \lim _{n \rightarrow \infty} c\left\|f_{n}\right\|_{\infty}=c\|f\|_{\infty},
$$

and the lemma is proved.

The proof of the theorem can now be completed. Since $\hat{I}$ is continuous and not identically zero, there is an open set $D$ on which $\hat{I}$ is bounded away from zero; say $|\hat{I}(\xi)| \geqslant h>0$ for $\xi \in D$. For any $\zeta$, let $f(x)=I(x) e^{-i x . \zeta}$. Then

$$
|\hat{f}(\xi)|=|\hat{I}(\xi-\zeta)| \geqslant h \quad \text { for } \xi \in D+\zeta \text {. }
$$

So $h^{2} \mu(D+\zeta)=\int_{D+\zeta} h^{2} d \mu \leqslant\left.\int\left|\hat{f}^{2} d \mu \leqslant c^{2} \sup _{I}\right| f\right|^{2}=c^{2}$, whence

$$
\sup \left\{\mu(D+\zeta): \zeta \in \mathbf{R}^{k}\right\} \leqslant \frac{c^{2}}{h^{2}}
$$

and $\mu$ is translation-bounded.

\section{Comment}

The paper [3] was concerned with a class of measures $\mu$ on $\mathbf{R}^{k}$ which satisfy the properties

(i) $\hat{A} \in L^{2}(\mu)$ for all $A \in \mathscr{B}_{0}$, and

(ii) if $\left(A_{n}\right)$ is a decreasing sequence of sets of $\mathscr{B}_{0}$ with empty intersection, then $\hat{A}_{n} \rightarrow 0$ in $L^{2}(\mu)$, 
and it was there shown that translation-bounded measures have both these properties. Thus it follows as a corollary to the theorem that (i) implies (ii) for Borel measures $\mu$.

\section{Acknowledgement}

We thank Wendy Robertson for her help and encouragement in the preparation of this paper.

\section{References}

[1] N. Dunford and J. T. Schwartz, Linear operators, part I: General theory (Interscience, New York, 1958).

[2] A. P. Robertson, 'Unconditional convergence and the Vitali-Hahn-Saks theorem', Colloque Anal. Function. [1971, Bordeaux] Bull. Soc. Math. France, Mémoire 31-32 (1972), 335-341.

[3] M. L. Thornett, 'A class of second-order stationary random measures', Stochastic Processes Appl. 8 (1979), 323-334.

Murdoch University

University of Western Australia

Murdoch, W.A. 6150

Nedlands, W.A. 6009

Australia

Australia 